Octave bandwidth hybrid-coupled microstrip diplexer for a broadband radio astronomy receiver

E. Villa, B. Aja, E. Artal, and L. de la Fuente

Citation: Review of Scientific Instruments 89, 064706 (2018); doi: 10.1063/1.5031885

View online: https://doi.org/10.1063/1.5031885

View Table of Contents: http://aip.scitation.org/toc/rsi/89/6

Published by the American Institute of Physics

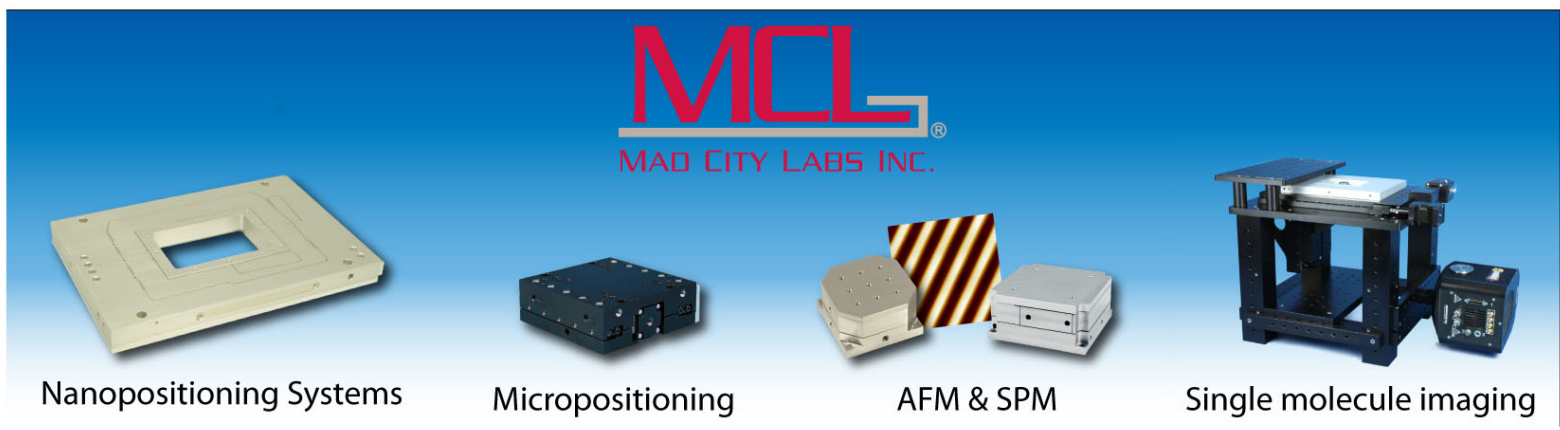




\title{
Octave bandwidth hybrid-coupled microstrip diplexer for a broadband radio astronomy receiver
}

\author{
E. Villa, ${ }^{1,2, a)}$ B. Aja, ${ }^{1}$ E. Artal, ${ }^{1}$ and L. de la Fuente ${ }^{1}$ \\ ${ }^{1}$ Departamento Ingeniería de Comunicaciones, Universidad de Cantabria, Plaza de la Ciencia s/n, \\ 39005 Santander, Spain \\ ${ }^{2}$ Instituto de Astrofísica de Canarias, IACTec, Vía Láctea s/n, 38205 La Laguna, Spain
}

(Received 1 April 2018; accepted 1 June 2018; published online 18 June 2018)

\begin{abstract}
A new octave bandwidth high selectivity compact microstrip diplexer working in the band from 10 to $20 \mathrm{GHz}$ is presented, intended to be a part of an electro-optical interferometer covering the whole frequency band. The circuit is based on the combination of hybrid couplers and bandpass filters both on the microstrip technology. The diplexer provides two output frequency bands which cover the 10-14 GHz (relative bandwidth of 33\%) and the 16-20 GHz (relative bandwidth of 22\%) ranges, respectively, with a stop band in between from 14 to $16 \mathrm{GHz}$. Measured results show a insertion loss level of $3.3 \mathrm{~dB}$ for both output bands and high selectivity performance, exhibiting a rejection level between output bands higher than $20 \mathrm{~dB}$ and return loss better than $10 \mathrm{~dB}$ in all ports. Published by AIP Publishing. https://doi.org/10.1063/1.5031885
\end{abstract}

\section{INTRODUCTION}

In many communication systems, in which a frequencyselective application is involved, specific subsystems are required to overcome the split of the signal into different subbands. Therefore, the use of multiplexers has been widely expounded to overwhelm this issue in transmitter or receiver systems, achieving high isolation between bands in order not to compromise the system performance. Moreover, the frequency multiplexing when wideband systems are involved demands a thorough knowledge of system configuration to select the most suitable alternative to compose it and properly operate. In this way, a receiver intended for radio astronomy applications usually covers great relative bandwidths; hence, the performance of each subsystem, which is part of it, is demanded to be extremely accurate and compliant with tight receiver specifications.

In this document, an octave bandwidth microwave diplexer to be part of a $10-20 \mathrm{GHz}$ receiver for radio astronomy applications is presented. This receiver is focused in the analysis of the early universe, through the study of the Cosmic Microwave Background (CMB) and the large-scale structure (LSS) of the universe. It is aimed to reveal the characteristics of the inflationary process responsible for the accelerated expansion of the early universe and the structure formation, which is currently observed. In this context, the data from the Planck ${ }^{1,2}$ and QUIJOTE ${ }^{3-5}$ experiments will be crucial to obtain a better knowledge of the foregrounds that cover the CMB. Moreover, the $10-20 \mathrm{GHz}$ receiver will also complement the measurements and data of the multifrequency instrument of the QUIJOTE project operating in the same frequency band but in a different topology. ${ }^{6}$ Therefore, in this receiver from 10 to $20 \mathrm{GHz}$, an electro-optical

a) Author to whom correspondence should be addressed: villae@unican.es interferometer is proposed to complement the latter data, and it is composed of two separate frequency bands $(10-14 \mathrm{GHz}$ and $16-20 \mathrm{GHz}$ ) to properly perform the microwave to optical frequency conversion.

Therefore, in order to split both frequency bands in the receiver, a microwave diplexer is used. The operation bandwidths of diplexer applications are quite different from few megahertz up to tens of gigahertz in their output bands, so many different topologies and technologies have been implemented. ${ }^{7}$ Microstrip solutions using band-stop filters, ${ }^{8}$ stubloaded configurations, ${ }^{9}$ or resonators ${ }^{10-12}$ have demonstrated significant performance. Yet, substrate integrated waveguide ${ }^{13}$ and waveguide designs ${ }^{14,15}$ have been competitive solutions to fulfill requirements.

Among above solutions, and intended for very wideband applications, waveguide implementations enable us to achieve outstanding results. ${ }^{14,15}$ However, the working frequency band in the presented receiver is a significant issue since covering an octave bandwidth with a standard rectangular waveguide makes it propagate higher-order modes in addition to the dominant mode that produce an erratic behavior in the proper operation of the design.

Considering the latter considerations, this manuscript proposes a novel octave bandwidth high selectivity compact microstrip diplexer based on the hybrid-coupled design with bandpass filters, properly defined to confine both the output frequency ranges and the selectivity between bands. The circuit covers the 10-20 GHz frequency band and splits it into a lower band from 10 to $14 \mathrm{GHz}$ and an upper band from 16 to $20 \mathrm{GHz}$, with a rejected band from 14 to $16 \mathrm{GHz}$. The document has five sections. This first section is an introduction, while the second one defines the receiver configuration in which the diplexer circuit is involved. Section III describes the design of the octave bandwidth diplexer. The experimental results are presented and discussed in Sec. IV, and finally, Sec. V draws general conclusions. 


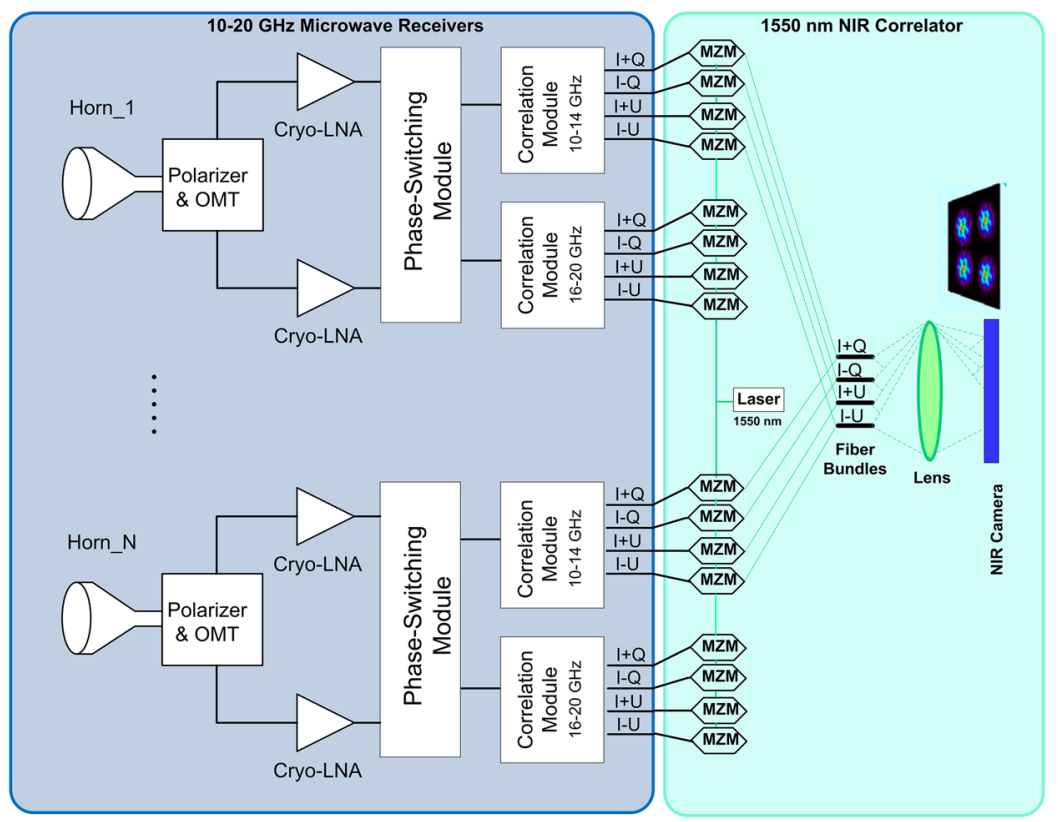

FIG. 1. Full interferometer configuration with the microwave (in blue) and optical (in light blue) parts.

\section{RECEIVER CONFIGURATION}

The receiver is an interferometer type that operates in the frequency band from 10 to $20 \mathrm{GHz}$, which combines very sensitive microwave instrumentation and a novel electrooptical correlator, which allows us to obtain, in the nearinfrared spectrum, the synthesized image of the Stokes parameters of the CMB polarization. A simplified overall receiver topology is shown in Fig. 1. The receiver configuration includes critical components, such as octave bandwidth feedhorn antenna, polarizer, orthomodes (OMT), or diplexers, as well as very low-noise amplifiers based on III-IV compound semiconductors, which all developments constitute technological challenges. The main advantage of the proposed correlator is its lower complexity with respect to classical correlator configurations.

Attending to the microwave receiver part, its configuration is depicted in Fig. 2. As stated before, it is composed of many broadband subsystems, which must cover the full bandwidth from 10 to $20 \mathrm{GHz}$ before the frequency separation in two subbands. In order to split the incoming bandwidth into the bands from 10 to $14 \mathrm{GHz}$ and from 16 to $20 \mathrm{GHz}$ and to avoid the undesired output frequency band from 14 to $16 \mathrm{GHz}$, a frequency selective circuit is used to perform the band separation: a diplexer. The performance of this unit is crucial in order to separate the frequency components for each band and, at the same time, to provide a high rejection of the undesired output band and high isolation between bands. The position of the diplexer in the receiver is shown in Fig. 2, located after the component separation of the incoming signal by the OMT and adequately amplified.

At the output connections of the Back-End Module (BEM) receiver, microwave correlation units are added in order to combine both microwave signals coming from separated bands from each branch. For each frequency band, an in-band microwave correlator with power splitters, fixed $90^{\circ}$ phase

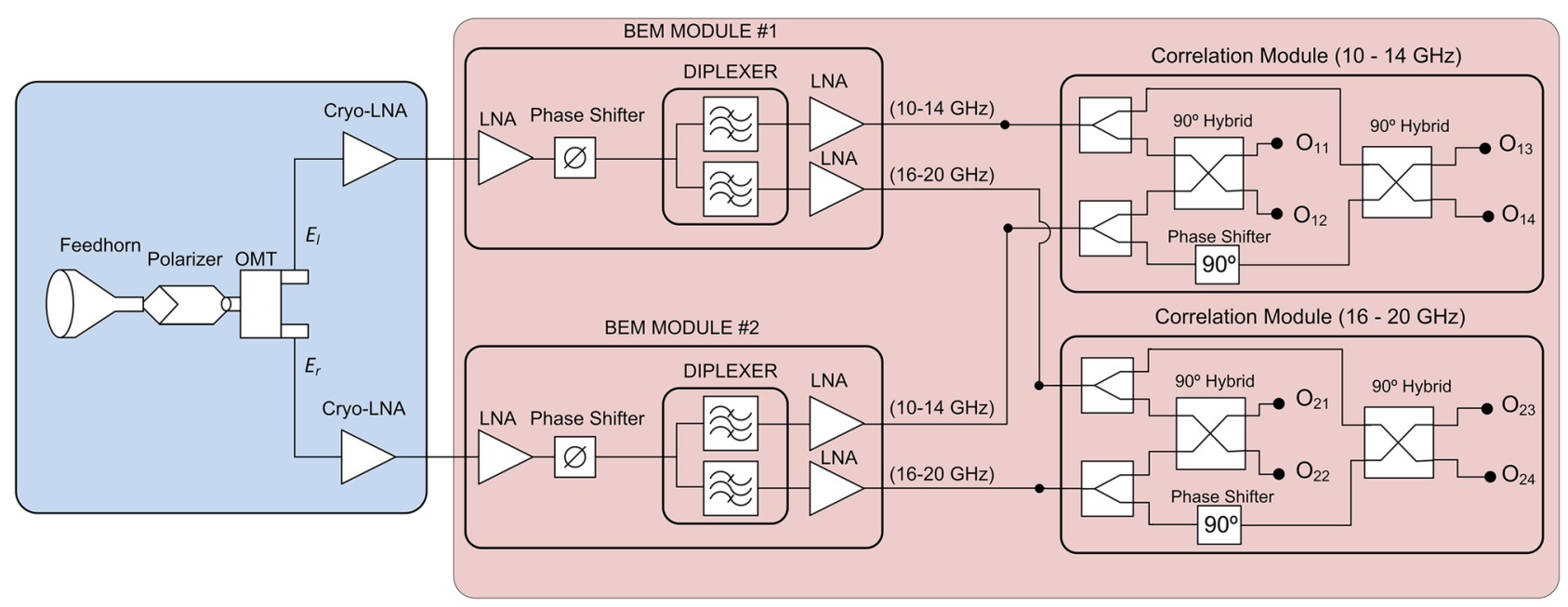

FIG. 2. $10-20 \mathrm{GHz}$ full receiver configuration (cryogenic part in blue and room temperature operation in red). 
shifter and $90^{\circ}$ hybrids, is placed. Then, the optical frequency conversion is performed to properly operate in the near-infrared frequency band.

\section{DIPLEXER CONCEPT AND DESIGN}

The diplexer is a key part of the wideband very sensitive receiver since it needs to split the signals into two subbands: 10-14 GHz and 16-20 GHz to subsequent signal correlation in each subband of the receiver, and to reject the $14-16 \mathrm{GHz}$ band in between.

The concept of the described diplexer is based on a balanced configuration using hybrid couplers in the main circuit branch and high selective bandpass filters to confine both output bands, in an improved topology to the design described in Ref. 15. The circuit schematic of the proposed diplexer is shown in Fig. 3. The proposed balanced topology avoids the use of a matching load termination in the isolated port of the output quadrature hybrid coupler when used in the diplexer, in combination with its high isolation. Moreover, this solution directly uses the isolated port of the input hybrid as one of the output bands. This effect is achieved by loading ports \#2 and \#3 of the input hybrid with reflective loads in the desired frequency band, which simultaneously provide a matching load effect for the other subband. Hence, no additional complex structures are needed at the isolated port of the hybrid. Besides, the great bandwidth covered could not be developed in a waveguide topology due the higher-order mode issue.
The combination of two $90^{\circ}$ hybrid couplers with two identical bandpass filters of one band, which are placed in between, makes the isolated port of the input hybrid propagate the rejected frequency components of the used filters. Then, a bandpass filter, designed in the other frequency band, confines the desired output band at the isolated port of the input coupler (see Fig. 3). Thus, the configuration for the diplexer shows that the upper frequency band is connected through the hybrid couplers, whereas the lower band is obtained in the isolated port of the input hybrid as shown in Fig. 3. Hence, the insertion loss level for both frequency bands is well balanced, which is significant to the whole system in order to accomplish its power budget.

\section{A. Diplexer analysis}

The analysis of the diplexer response is performed using the schematic shown in Fig. 4. The different reflection coefficients at each subsystem ports are considered, in order to evaluate each frequency band of the diplexer taking into account the mismatching between subsystems.

The diplexer is analyzed obtaining the gain for each transmitted signal from input port \#1 to output ports \#2 and \#3. A good isolation level in the couplers entails a negligible influence of the isolated port (\#H4 of the input coupler in Fig. 4) for the transmission signal through output port \#2. Then, a significant isolation of the couplers together with a good matching in their ports enable to calculate the gain of the whole balanced network from input port \#1 and output port \#2 considering all the reflections coefficients shown in Fig. 4. Besides, the
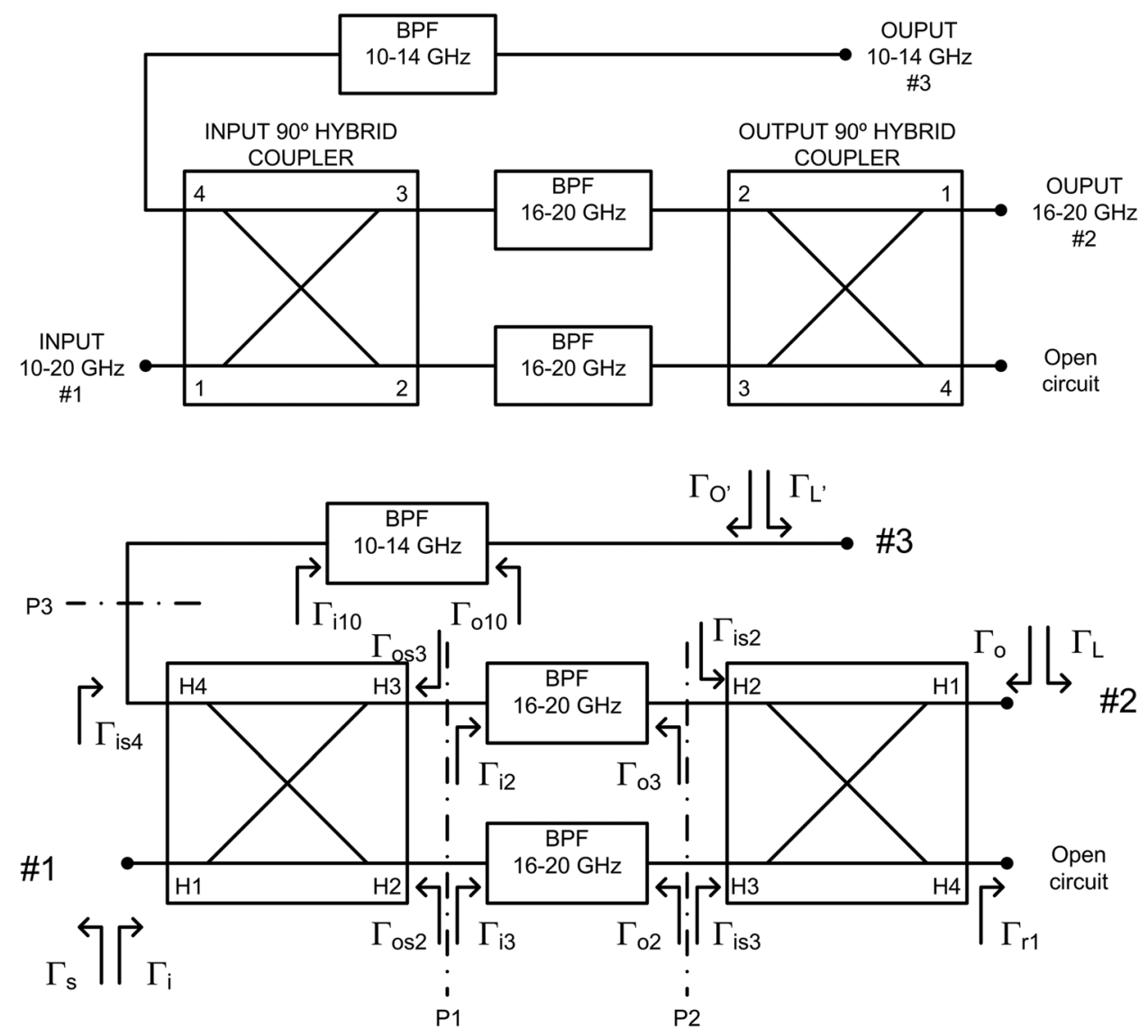

FIG. 3. Circuit schematic of the proposed diplexer.
FIG. 4. Circuit configuration of the diplexer considering the reflection coefficients for its analysis. 
transmission coefficients of the 16-20 GHz bandpass filters are also considered in the calculation, whilst a reflection coefficient is shown in the isolated ports of the hybrid couplers $\left(\Gamma_{\mathrm{r} 1}\right.$ and $\left.\Gamma_{\mathrm{i} 10}\right)$. This analysis of the diplexer for the $16-20 \mathrm{GHz}$ band takes into account that the isolated port of both hybrid couplers is loaded with a reflective open end, which means $\Gamma_{\mathrm{r} 1}=1$, and, indeed, it makes easier the circuit manufacture since $50 \Omega$ loads are avoided, and the performance of the balanced configuration is not affected.

In a similar way, the transmission signal to output port \#3 is analyzed as the transfer gain of the input hybrid coupler (between its \#H1 and \#H4 ports) and the transfer gain of the bandpass filter of the $10-14 \mathrm{GHz}$ band. Moreover, the mismatching coefficient between the subsystems at its interface (reference plane P3 in Fig. 4) is considered to correct the losses due to those mismatchings.

In the light of the above, the analysis is presented in terms of the cascaded transfer gain of the different subsystems corrected by the mismatching effect between them. Therefore, the transfer gain between ports \#1 and \#2 can be expressed as

$$
G_{T 1-2}=\left|S_{21}\right|^{2},
$$

where $S_{21}$ is the whole gain of the balanced structure from port \#1 to port \#2 in which each of the $16-20 \mathrm{GHz}$ filters is located. As all the subsystems are the same and symmetrical, $S_{21}$ is defined as

$$
\begin{aligned}
S_{21}= & \frac{\mathrm{S}_{12-\mathrm{BPF} 16-20} \cdot \mathrm{S}_{\mathrm{H} 1 \mathrm{H} 2} \cdot \mathrm{S}_{\mathrm{H} 1 \mathrm{H} 3} \cdot\left(-\Gamma_{\mathrm{i} 2} \cdot \Gamma_{\mathrm{i} 10} \cdot \mathrm{S}_{\mathrm{H} 1 \mathrm{H} 2}{ }^{2}+\Gamma_{\mathrm{i} 2} \cdot \Gamma_{\mathrm{i} 10} \cdot \mathrm{S}_{\mathrm{H} 1 \mathrm{H} 3}{ }^{2}+1\right)}{\left(\Gamma_{\mathrm{is} 3} \cdot \mathrm{S}_{11-\mathrm{BPF} 16-20}-1\right) \cdot \mathrm{A}} \\
& +\frac{\mathrm{S}_{12-\mathrm{BPF} 16-20} \cdot \mathrm{S}_{\mathrm{H} 1 \mathrm{H} 3} \cdot \mathrm{S}_{\mathrm{H} 1 \mathrm{H} 2} \cdot\left(\Gamma_{\mathrm{i} 3} \cdot \Gamma_{\mathrm{i} 10} \cdot \mathrm{S}_{\mathrm{H} 1 \mathrm{H} 2}{ }^{2}+\Gamma_{\mathrm{i} 3} \cdot \Gamma_{\mathrm{i} 10} \cdot \mathrm{S}_{\mathrm{H} 1 \mathrm{H} 3}{ }^{2}+1\right)}{\left(\Gamma_{\mathrm{is} 2} \cdot \mathrm{S}_{11-\mathrm{BPF} 16-20}{ }^{2}-1\right) \cdot \mathrm{A}},
\end{aligned}
$$

where $S_{i j-B P F 16-20}$ and $\mathrm{S}_{\mathrm{ii}-\mathrm{BPF} 16-20}$ are the scattering parameters of the 16-20 GHz bandpass filter, $\mathrm{S}_{\mathrm{HiHj}}$ and $\mathrm{S}_{\mathrm{HiHi}}$ are the scattering parameters of the hybrid couplers, and the parameter A is defined as

$$
\mathrm{A}=\Gamma_{\mathrm{i} 2} \cdot \Gamma_{\mathrm{i} 10} \cdot \mathrm{S}_{\mathrm{H} 1 \mathrm{H} 2}{ }^{2}+\Gamma_{\mathrm{i} 3} \cdot \Gamma_{\mathrm{i} 10} \cdot \mathrm{S}_{\mathrm{H} 1 \mathrm{H} 3}{ }^{2}-1 .
$$

Moreover, the different reflection coefficients included in Eq. (2) are defined as

$$
\Gamma_{O S 3}=\mathrm{S}_{\mathrm{H} 3 \mathrm{H} 3}+\frac{\mathrm{S}_{\mathrm{H} 3 \mathrm{H} 2} \cdot \mathrm{S}_{\mathrm{H} 2 \mathrm{H} 3} \cdot \Gamma_{\mathrm{i} 3}}{1-\mathrm{S}_{\mathrm{H} 2 \mathrm{H} 2} \cdot \Gamma_{\mathrm{i} 3}}+\frac{\mathrm{S}_{\mathrm{H} 4 \mathrm{H} 3} \cdot \mathrm{S}_{\mathrm{H} 3 \mathrm{H} 4} \cdot \Gamma_{\mathrm{i} 10}}{1-\mathrm{S}_{\mathrm{H} 4 \mathrm{H} 4} \cdot \Gamma_{\mathrm{i} 10}},
$$

$$
\Gamma_{O S 2}=\mathrm{S}_{\mathrm{H} 2 \mathrm{H} 2}+\frac{\mathrm{S}_{\mathrm{H} 3 \mathrm{H} 2} \cdot \mathrm{S}_{\mathrm{H} 2 \mathrm{H} 3} \cdot \Gamma_{\mathrm{i} 2}}{1-\mathrm{S}_{\mathrm{H} 2 \mathrm{H} 2} \cdot \Gamma_{\mathrm{i} 2}}+\frac{\mathrm{S}_{\mathrm{H} 4 \mathrm{H} 2} \cdot \mathrm{S}_{\mathrm{H} 2 \mathrm{H} 4} \cdot \Gamma_{\mathrm{i} 10}}{1-\mathrm{S}_{\mathrm{H} 4 \mathrm{H} 4} \cdot \Gamma_{\mathrm{i} 10}},
$$

$\Gamma_{i 2}=\mathrm{S}_{11-\mathrm{BPF} 16-20}+\frac{\mathrm{S}_{21-\mathrm{BPF} 16-20} \cdot \mathrm{S}_{12-\mathrm{BPF} 16-20} \cdot \Gamma_{\mathrm{iS} 2}}{1-\mathrm{S}_{22-\mathrm{BPF} 16-20} \cdot \Gamma_{\mathrm{iS} 2}}$,

$\Gamma_{i 3}=\mathrm{S}_{11-\mathrm{BPF} 16-20}+\frac{\mathrm{S}_{21-\mathrm{BPF} 16-20} \cdot \mathrm{S}_{12-\mathrm{BPF} 16-20} \cdot \Gamma_{\mathrm{iS} 3}}{1-\mathrm{S}_{22-\mathrm{BPF} 16-20} \cdot \Gamma_{\mathrm{iS} 3}}$,

$$
\begin{gathered}
\Gamma_{i S 2}=\mathrm{S}_{\mathrm{H} 2 \mathrm{H} 2}+\frac{\mathrm{S}_{\mathrm{H} 4 \mathrm{H} 2} \cdot \mathrm{S}_{\mathrm{H} 2 \mathrm{H} 4} \cdot \Gamma_{\mathrm{r} 1}}{1-\mathrm{S}_{\mathrm{H} 4 \mathrm{H} 4} \cdot \Gamma_{\mathrm{r} 1}}, \\
\Gamma_{i S 3}=\mathrm{S}_{\mathrm{H} 3 \mathrm{H} 3}+\frac{\mathrm{S}_{\mathrm{H} 4 \mathrm{H} 3} \cdot \mathrm{S}_{\mathrm{H} 3 \mathrm{H} 4} \cdot \Gamma_{\mathrm{r} 1}}{1-\mathrm{S}_{\mathrm{H} 4 \mathrm{H} 4} \cdot \Gamma_{\mathrm{r} 1}}, \\
\Gamma_{O 2}=\mathrm{S}_{22-\mathrm{BPF} 16-20}+\frac{\mathrm{S}_{12-\mathrm{BPF} 16-20} \cdot \mathrm{S}_{21-\mathrm{BPF} 16-20} \cdot \Gamma_{\mathrm{OS} 2}}{1-\mathrm{S}_{11-\mathrm{BPF} 16-20} \cdot \Gamma_{\mathrm{OS} 2}}, \\
\Gamma_{O 3}=\mathrm{S}_{22-\mathrm{BPF} 16-20}+\frac{\mathrm{S}_{12-\mathrm{BPF} 16-20} \cdot \mathrm{S}_{21-\mathrm{BPF} 16-20} \cdot \Gamma_{\mathrm{OS} 3}}{1-\mathrm{S}_{11-\mathrm{BPF} 16-20} \cdot \Gamma_{\mathrm{OS} 3}} .
\end{gathered}
$$

In an analogous way, considering ports \#1 and \#3, the transfer gain is calculated as

$$
G_{T 1-3}=\frac{G_{T H 1-4} \cdot G_{T 10-14}}{M_{3}},
$$

where $G_{T H 1-4}$ is the transfer gain of the input hybrid coupler, $G_{T 10-14}$ is the transfer gain of the 10-14 bandpass filter, and $M_{3}$ is the mismatching coefficient at reference plane P3. The coefficients for each variable of the above equation are expressed as

$$
\mathrm{G}_{T H 1-4}=\frac{\left|\mathrm{S}_{\mathrm{H} 41}\right|^{2} \cdot\left(1-\left|\Gamma_{\mathrm{S}}\right|^{2}\right) \cdot\left(1-\left|\Gamma_{\mathrm{i} 10}\right|^{2}\right)}{\left|1-\Gamma_{\mathrm{i}} \cdot \Gamma_{\mathrm{S}}\right|^{2} \cdot\left|1-\mathrm{S}_{\mathrm{H} 4 \mathrm{H} 4} \cdot \Gamma_{\mathrm{i} 10}\right|^{2}}=\frac{\left|S_{H 4 H 2} \cdot S_{H 2 H 1} \cdot \Gamma_{\mathrm{i} 3}+S_{H 4 H 3} \cdot S_{H 3 H 1} \cdot \Gamma_{\mathrm{i} 2}\right|^{2} \cdot\left(1-\left|\Gamma_{\mathrm{S}}\right|^{2}\right) \cdot\left(1-\left|\Gamma_{\mathrm{i} 10}\right|^{2}\right)}{\left|1-\Gamma_{\mathrm{i}} \cdot \Gamma_{\mathrm{S}}\right|^{2} \cdot\left|1-\mathrm{S}_{\mathrm{H} 4 \mathrm{H} 4} \cdot \Gamma_{\mathrm{i} 10}\right|^{2}},
$$

$$
\begin{aligned}
\mathrm{G}_{T 10-14} & =\frac{\left|\mathrm{S}_{21-B P F 10-14}\right|^{2} \cdot\left(1-\left|\Gamma_{\mathrm{iS} 4}\right|^{2}\right) \cdot\left(1-\left|\Gamma_{\mathrm{L}^{\prime}}\right|^{2}\right)}{\left|1-\Gamma_{\mathrm{i} 10} \cdot \Gamma_{\mathrm{iS} 4}\right|^{2} \cdot\left|1-\mathrm{S}_{22-\mathrm{BPF} 10-14} \cdot \Gamma_{\mathrm{L}^{\prime}}\right|^{2}}, \\
\mathrm{M}_{3} & =\frac{\left(1-\left|\Gamma_{\mathrm{iS} 4}\right|^{2}\right) \cdot\left(1-\left|\Gamma_{\mathrm{i} 10}\right|^{2}\right)}{\left|1-\Gamma_{\mathrm{iS} 4} \cdot \Gamma_{\mathrm{i} 10}\right|^{2}},
\end{aligned}
$$

where $S_{\mathrm{ij}-\mathrm{BPF} 10-14}$ and $\mathrm{S}_{\mathrm{ii}-\mathrm{BPF} 10-14}$ are the scattering parameters of the 10-14 GHz bandpass filter.

Replacing in Eq. (12) and considering the matching loads at each port, the transfer gain for the low-frequency band is obtained as 


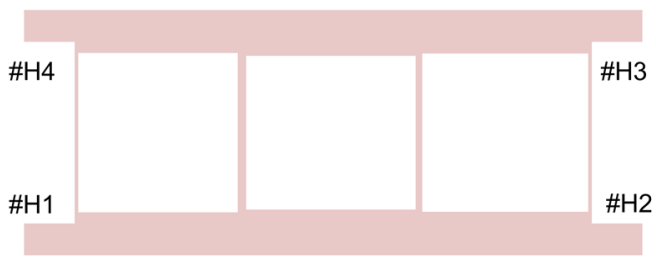

a)

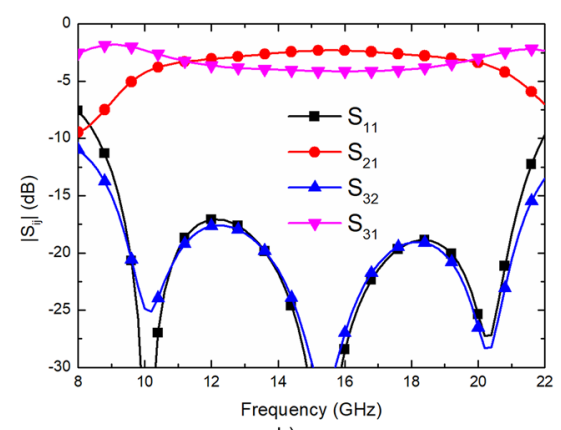

b)
FIG. 5. Electromagnetic simulation of the quadrature hybrid coupler in terms of the transmission, isolation, and matching parameters. (a) Layout of the hybrid coupler. (b) Scattering parameters.

$$
G_{T 1-3}=\frac{\left|S_{H 4 H 2} \cdot S_{H 2 H 1} \cdot \Gamma_{\mathrm{i} 3}+S_{H 4 H 3} \cdot S_{H 3 H 1} \cdot \Gamma_{\mathrm{i} 2}\right|^{2} \cdot\left|S_{21-B P F-10-14}\right|^{2}}{\left|1-S_{\mathrm{H} 4 \mathrm{H} 4} \cdot \Gamma_{\mathrm{i} 10}\right|^{2}},
$$

where the load condition $\Gamma_{i 10}=\mathrm{S}_{11-\mathrm{BPF} 10-14}$ is assumed.

\section{B. $90^{\circ}$ quadrature hybrid coupler}

The design of the $90^{\circ}$ hybrid couplers is based on a 3 -section synthesis ${ }^{16,17}$ to properly cover the $10-20 \mathrm{GHz}$ frequency band in the microstrip technology, considering a symmetrical configuration. The use of $90^{\circ}$ hybrid coupler involves competitive manufacturing and assembly improvements facing approaches such as Lange, coupled-line, or overlay couplers since physical dimensions or interconnections between elements are not critical issues in the design and manufacture procedure. Figure 5 depicts the electromagnetic simulation, performed with HFSS 3D software, of the $90^{\circ}$ hybrid coupler, showing the matching of the input port (port \#H1 in Fig. 4 for the coupler), the transmission losses for both outputs (ports \#H2 and \#H3 in Fig. 4), and the isolation between outputs. Besides, the phase difference performance between both outputs (ports \#H2 and \#H3 in Fig. 4) is shown in Fig. 6, with an in-band average phase difference of $89.3^{\circ}$.

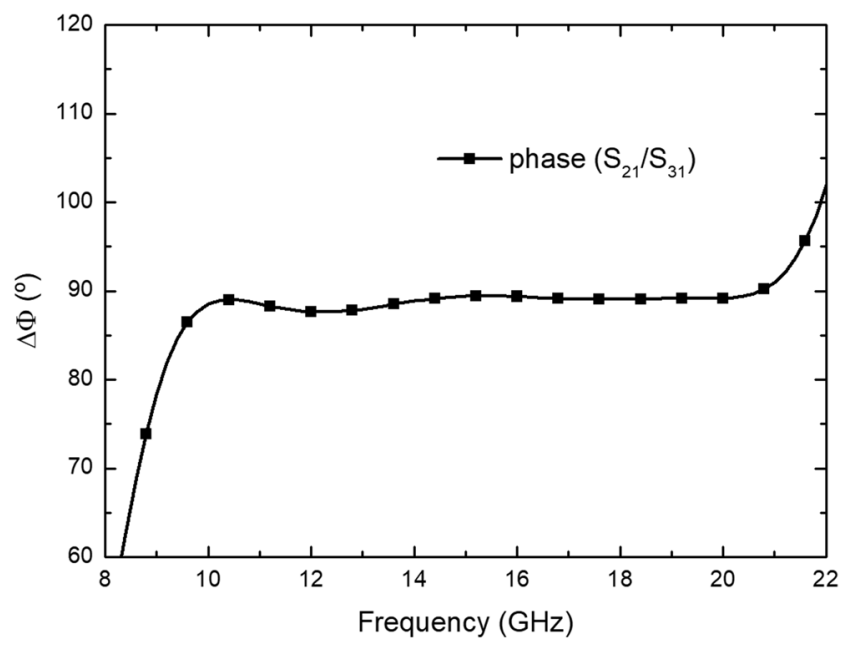

FIG. 6. Electromagnetic simulation of the quadrature hybrid coupler in terms of its phase performance.

\section{Bandpass filters}

Both bandpass filters (10-14 GHz and 16-20 GHz bands) are designed using a shunt stub configuration in microstrip lines. ${ }^{17}$ Both of them have to highly reject the other subband of the diplexer outputs, in order to avoid any undesired frequency component. In order to fulfill the bandwidth and rejection requirements, both filters are designed using $5 \mathrm{sec}-$ tions with open-end stubs in each one. Both bandpass filter layouts are shown in Fig. 7, and Fig. 8 shows the electromagnetic simulations, also with HFSS, of both bandpass filters, in terms of their insertion loss and input reflection coefficient.

\section{EXPERIMENTAL CHARACTERIZATION}

The diplexer has been manufactured on a $0.254-\mu \mathrm{m}$ thick CLTE-XT substrate $\left(\varepsilon_{\mathrm{r}}=2.89\right)$ from Rogers Corporation. It has been assembled in a chassis, specifically machined for integration purposes, provided with commercial superSMA jack connectors, model 214-500SF from Southwest Microwave, to properly cover the full bandwidth. Figure 9 shows a photograph of the diplexer assembly inside the chassis.

The performance of the diplexer has been measured using a vector network analyzer through a short-open-load-through standard calibration at the coaxial ports. The measured and simulated transmission coefficients of both outputs and the isolation between them are shown in Fig. 10, while the return loss of each port (input and both outputs) is depicted in Fig. 11. The overall response of the diplexer shows return loss better than $13 \mathrm{~dB}$, and this value is reduced to around $10 \mathrm{~dB}$ in a narrow frequency band, around $10 \mathrm{GHz}$ and $13 \mathrm{GHz}$, which is due to the combination of the hybrid coupler with the bandpass filters. This return loss achieved in the measurement is considered enough in order not to severely affect the whole system. These results are compared to the simulation results (empty symbols) provided by a HFSS electromagnetic simulator. Average in-band insertion of loss of around $2.3 \mathrm{~dB}$ is obtained in simulation, whereas around $3.3 \mathrm{~dB}$ in the measurement. The slight difference between these values is due 


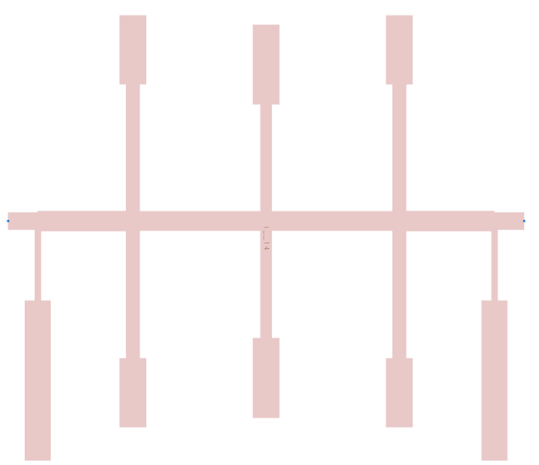

a)

to the additional losses provided by the connectors and the coaxial-to-microstrip transitions implemented in the chassis assembly and not considered in the simulation. Besides, the SMA coaxial port calibration enables to characterize the circuit in a similar way to the integration in the whole system, since the diplexer will be connected to other subsystems through coaxial connectors, and a real estimation of its insertion loss is provided. The rejection measured in each subband shows levels above $23 \mathrm{~dB}$ over the whole frequency bands.

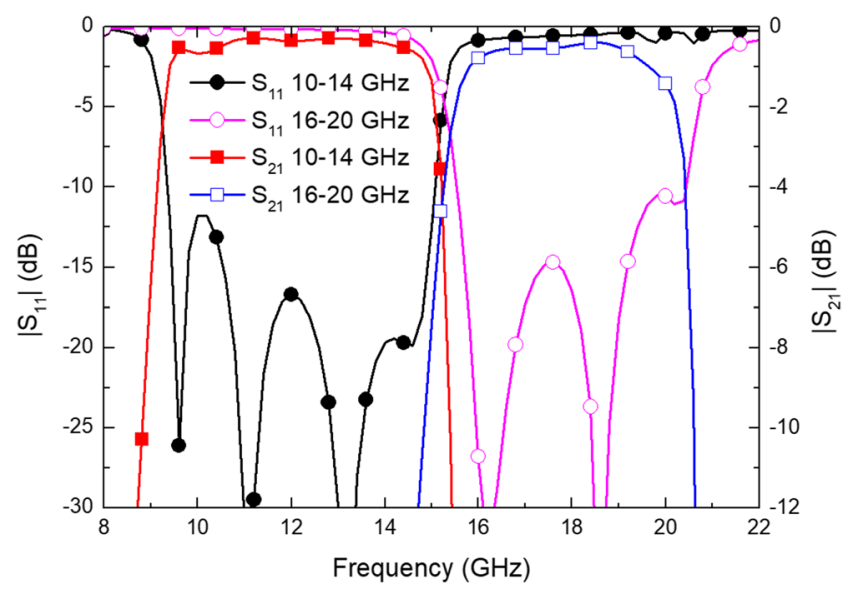

FIG. 8. Electromagnetic simulations of the $10-14 \mathrm{GHz}$ and $16-20 \mathrm{GHz}$ bandpass filters.

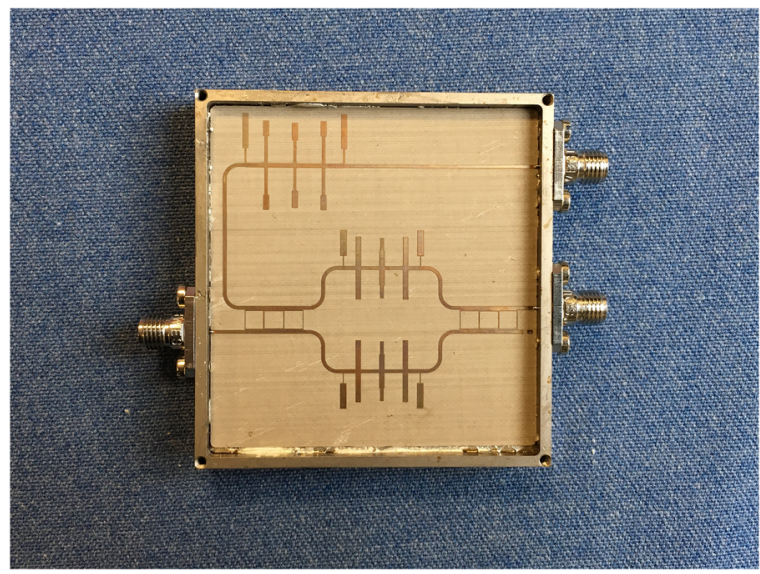

FIG. 9. Picture of the manufactured diplexer assembled in a chassis with super-SMA connectors (size: $61 \times 57.7 \mathrm{~mm}^{2}$ ).
The performance of the diplexer is listed in Table I, which also shows the performance of the different designs presented in the literature. The comparison between the described diplexer and other proposals validates the performance obtained in this paper as a competitive alternative to

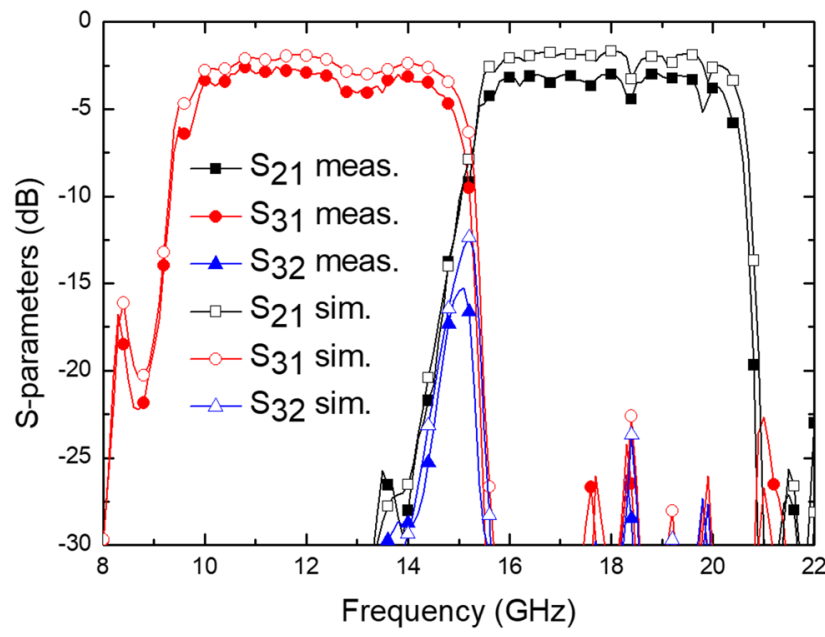

FIG. 10. Simulated (empty symbols) and measured (filled symbols) performance of the diplexer: insertion loss of each subband and isolation between them.

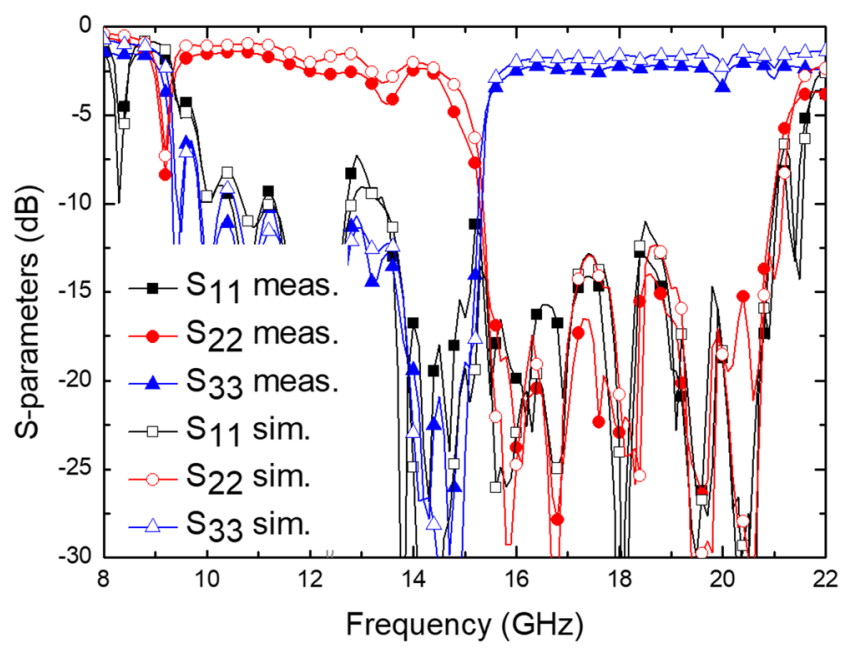

FIG. 11. Simulated (empty symbols) and measured (filled symbols) performance of the diplexer: matching results at each port. 
TABLE I. Performance comparison of the presented work with other diplexers.

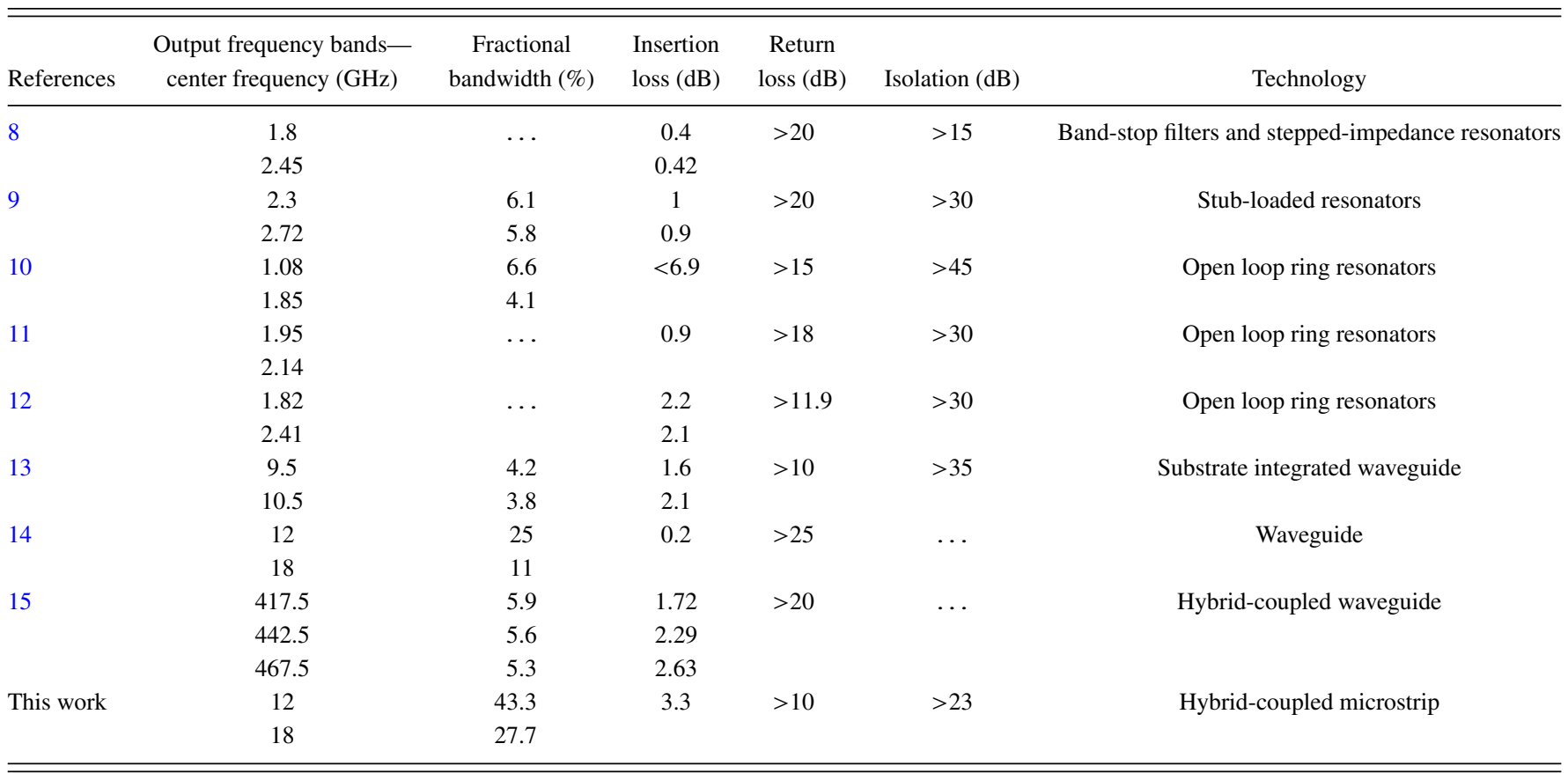

cover great bandwidths with high isolation values between undesired frequency bands.

\section{CONCLUSIONS}

A new octave bandwidth microstrip diplexer intended for a radio astronomy receiver has been described. The design uses a balanced topology with the combination of two $90^{\circ}$ hybrid couplers and three bandpass filters to provide one output band from 16 to $20 \mathrm{GHz}$, while the other from 10 to $14 \mathrm{GHz}$ is obtained through the rejected signal at the isolated port of the first hybrid coupler. The presented configuration avoids the use of a matching load in the isolated port of the $90^{\circ}$ hybrid coupler. The diplexer designed has been assembled in a chassis with coaxial ports for connectivity purposes. The two wideband output bands, 10-14 GHz and 16-20 GHz, have been properly split, with average insertion loss of around $3.3 \mathrm{~dB}$ and isolation between diplexer outputs higher than $23 \mathrm{~dB}$. The performance of the described diplexer validates its use on an interferometer type radio astronomy receiver to cover the full $10-20 \mathrm{GHz}$ frequency band.

\section{ACKNOWLEDGMENTS}

This work has been funded by the Spanish Ministry of Economy, Industry and Competitiveness under Grant No. ESP2015-70646-C2-2-R.

${ }^{1}$ M. Bersanelli et al., Astron. Astrophys. 520, A4 (2010).

${ }^{2}$ N. Aghanim et al., Astron. Astrophys. 571, A3 (2014).

${ }^{3}$ J. A. Rubiño-Martin, R. Rebolo, M. Tucci, R. Génova-Santos, S. R. Hildebrandt, R. Hoyland, J. M. Herreros, F. Gómez-Renasco, C. López, E. Martínez-González, P. Vielva, D. Herranz, F. J. Casas, E. Artal, B. Aja, L. de la Fuente, J. L. Cano, E. Villa, A. Mediavilla, J. P. Pascual, L. Piccirillo, B. Maffei, G. Pisano, R. A. Watson, R. Davis, R. Davies, R. Battye, R. Saunders, K. Grainge, P. Scott, M. Hobson, A. Lasenby, G. Murga,
C. Gómez, A. Gómez, J. Arino, R. Sanquirce, J. Pan, A. Vizcarguenaga, and B. Etxeita, in Highlights of Spanish Astrophysics V, Astrophysics and Space Science Proceedings (Springer, Berlin, 2010), Part 3, pp. 127-135.

${ }^{4}$ M. López-Caniego, R. Rebolo, M. Aguiar, R. Génova-Santos, F. GómezReñasco, C. Gutierrez, J. M. Herreros, R. J. Hoyland, C. López-Caraballo, A. E. Pelaez Santos, F. Poidevin, J. A. Rubiño-Martín, V. Sanchez de la Rosa, D. Tramonte, A. Vega-Moreno, T. Viera-Curbelo, R. Vignaga, E. Martínez-González, R. B. Barreiro, B. Casaponsa, F. J. Casas, J. M. Diego, R. Fernández-Cobos, D. Herranz, D. Ortiz, P. Vielva, E. Artal, B. Aja, J. Cagigas, J. L. Cano, L. de la Fuente, A. Mediavilla, J. V. Terán, E. Villa, L. Piccirillo, R. Battye, E. Blackhurst, M. Brown, R. D. Davies, R. J. Davis, C. Dickinson, K. Grainge, S. Harper, B. Maffei, M. McCulloch, S. Melhuish, G. Pisano, R. A. Watson, M. Hobson, A. Lasenby, R. Saunders, and P. Scott, "Instrumentation and methods for astrophysics," in Conference Proceedings Rencontres du Vietnam 2013: Cosmology in the Planck Era (ICISE, 2014), p. 1.

${ }^{5}$ E. Villa, J. L. Cano, J. Cagigas, D. Ortiz, F. J. Casas, A. R. Pérez, B. Aja, J. V. Terán, L. de la Fuente, E. Artal, R. Hoyland, and A. Mediavilla, Rev. Sci. Instrum. 86, 024702 (2015).

${ }^{6}$ R. J. Hoyland, M. Aguiar-González, B. Aja, J. Ariño, E. Artal, R. B. Barreiro, E. J. Blackhurst, J. Cagigas, J. L. Cano de Diego, F. J. Casas, R. J. Davis, C. Dickinson, B. E. Arriaga, R. Fernandez-Cobos, L. de la Fuente, R. Génova-Santos, A. Gómez, C. Gomez, F. Gómez-Reñasco, K. Grainge, S. Harper, D. Herran, J. M. Herreros, G. A. Herrera, M. P. Hobson, A. N. Lasenby, M. Lopez-Caniego, C. López-Caraballo, B. Maffei, E. Martinez-Gonzalez, M. McCulloch, S. Melhuish, A. Mediavilla, G. Murga, D. Ortiz, L. Piccirillo, G. Pisano, R. Rebolo-López, J. A. RubiñoMartin, J. Luis Ruiz, V. Sanchez de la Rosa, R. Sanquirce, A. Vega-Moreno, P. Vielva, T. Viera-Curbelo, E. Villa, A. Vizcargüenaga, and R. A. Watson, Proc. SPIE 8452, 845233 (2012).

${ }^{7}$ R. J. Cameron and M. Yu, IEEE Microwave Mag. 8, 46 (2007).

${ }^{8}$ D. H. N. Bui, T. P. Vuong, B. Allard, J. Verdier, and P. Benech, Electron. Lett. 53, 552 (2017).

${ }^{9}$ F. Huang, J. Wang, L. Zhu, and W. Wu, Electron. Lett. 52, 1994 (2016).

${ }^{10}$ W. Feng, Y. Zhang, and W. Che, IEEE Trans. Circuits Syst. II: Express Briefs 64, 1047 (2016).

${ }^{11} \mathrm{X}$. Guan, W. Huang, H. Liu, P. Wen, and Q. Cheng, in IEEE International Workshop on Electromagnetics: Applications and Student Innovation Competition, Nanjing, China, 16-18 May 2016 (IEEE, 2016), pp. $1-3$.

${ }^{12}$ J. M. Yan, H. Y. Zhou, and L. Z. Cao, Electron. Lett. 52, 1613 (2016). 
${ }^{13}$ S. Sirci, J. D. Martinez, J. Vague, and V. E. Boria, IEEE Microwave Wireless Compon. Lett. 25, 430 (2015).

${ }^{14}$ F. Teberio, I. Arregui, M. Guglielmi, A. Gomez-Torrent, P. Soto, M. A. G. Laso, and V. E. Boria, in IEEE MTT-S International Microwave Symposium, San Francisco, United States, 22-27 May 2016 (IEEE, 2016), pp. $1-4$.
${ }^{15}$ T. Kojima, A. Gonzalez, S. Asayama, and Y. Uzama, IEEE Trans. Terahertz Sci. Technol. 7, 10 (2017).

${ }^{16}$ R. Levy and L. F. Lind, IEEE Trans. Microwave Theory Tech. 16, 80 (1968).

${ }^{17}$ G. L. Matthaei, L. Young, and E. M. T. Jones, Microwave Filters, Impedance Matching Networks and Coupling Structures (McGraw-Hill, New York, 1964). 